Cite this: Phys. Chem. Chem. Phys., 2013, 15, 17672

Received 3rd April 2013, Accepted 28th August 2013

DOI: $10.1039 / \mathrm{c} 3 \mathrm{cp} 51399 \mathrm{~h}$

www.rsc.org/pccp

\title{
UVA irradiation of fatty acids and their oxidized products substantially increases their ability to generate singlet oxygen
}

\author{
Johannes Regensburger,* Tim Maisch, Alena Knak, Anita Gollmer, \\ Ariane Felgentraeger, Karin Lehner and Wolfgang Baeumler
}

\begin{abstract}
UVA radiation plays an important role for adverse reactions in human tissue. UVA penetrates epidermis and dermis of skin being absorbed by various biomolecules, especially endogenous photosensitizers. This may generate deleterious singlet oxygen $\left({ }^{1} \mathrm{O}_{2}\right)$ that oxidizes fatty acids in cell membranes, lipoproteins, and other lipid-containing structures such as the epidermal barrier. Indications exist that fatty acids are not only the target of ${ }^{1} \mathrm{O}_{2}$ but also act as potential photosensitizers under UVA irradiation, if already oxidized. Five different fatty acids in ethanol solution (stearic, oleic, linoleic, linolenic and arachidonic acid) were exposed to UVA radiation ( $355 \mathrm{~nm}, 100 \mathrm{~mW}$ ) for 30 seconds. ${ }^{1} \mathrm{O}_{2}$ luminescence was detected time-resolved at $1270 \mathrm{~nm}$ and confirmed in spectrally-resolved experiments. The more double bonds fatty acids have the more ${ }^{1} \mathrm{O}_{2}$ photons were detected. In addition, fatty acids were continuously exposed to broadband UVA for up to $240 \mathrm{~min}$. During that time span, UVA absorption and ${ }^{1} \mathrm{O}_{2}$ luminescence substantially increased with irradiation time, reached a maximum and decreased again. HPLC-MS analysis showed that the amount of peroxidized fatty acids and the ${ }^{1} \mathrm{O}_{2}$ generation increased and decreased in parallel. This indicates the high potential of peroxidized fatty acids to produce ${ }^{1} \mathrm{O}_{2}$ under UVA irradiation. In conclusion, fatty acids along with peroxidized products are weak endogenous photosensitizers but become strong photosensitizers under continuous UVA irradiation. Since fatty acids and their oxidized products are ubiquitous in living cells and in skin, which is frequently and long-lasting exposed to UVA radiation, this photosensitizing effect may contribute to initiation of deleterious photooxidative processes in tissue.
\end{abstract}

\section{Introduction}

Solar radiation on earth comprises UVA $(\sim 95 \%)$ and UVB $(\sim 5 \%)$ radiation. UV radiation can induce DNA damage, oxidative stress or immune-suppression. ${ }^{1} \mathrm{UV}$ radiation affects cellular and tissue integrity $^{2-10}$ and is a major cause of human skin cancer. ${ }^{11,12}$ Furthermore, statistical investigations in USA provide evidence that skin cancer is the most frequent diagnosed cancer in humans (40\% of all cancer diseases). ${ }^{13}$ Due to a change of lifestyle and an increased expectation of life, exposure to UVA-radiation should even have a higher impact on skin damage. ${ }^{14}$

UVA-radiation (320-400 $\mathrm{nm}$ ) deeply penetrates skin and can be absorbed by various endogenous molecules. In case that such an endogenous molecule represents a photosensitizer, energy or charge can be transferred via an excited triplet state to oxygen. Energy transfer may excite an oxygen molecule in its triplet ground state to its first singlet state (singlet oxygen).

Department of Dermatology, University of Regensburg, 93042 Regensburg, Germany. E-mail: johannes.regensburger@klinik.uni-regensburg.de; Fax: +49-941-944-8943; Tel: +49-941-944-8944
Charge transfer may generate other reactive oxygen species such as radicals. All these species are frequently responsible for deleterious cellular effects, in which singlet oxygen plays a major role. ${ }^{15-17}$

Singlet oxygen is intentionally produced in photodynamic therapy to kill cancer cells via photooxidation of cellular constituents. In the case of UVA radiation, it has already been shown that keratinocytes or living skin can generate singlet oxygen in skin without an additional exogenous photosensitizers. ${ }^{18}$ In that case, photosensitizers are endogenous cell components like flavines, lipids or oxidized fatty acids. ${ }^{19,20}$ Lipids and fatty acids are ubiquitous inside skin cells and also outside cells in the epidermis.

We already detected the luminescence of singlet oxygen, which was generated by different fatty acids and lipids in solution. ${ }^{20}$ The signal intensities from these molecules are usually low and it remains questionable whether and to what extent singlet oxygen generation by fatty acids and lipids might contribute to deleterious effects of UVA on skin. A reason for the small signal was the low absorption of UVA radiation in fatty acids or lipids. However, the structure of such endogenous photosensitizers 
may change under UVA irradiation, which may in turn change the UV absorption and hence singlet oxygen production.

In the present investigation, we exposed different fatty acids (stearic, oleic, linoleic, linolenic and arachidonic acid), which are main constituents of cell lipids, ${ }^{21,22}$ to UVA radiation, the change in absorption was measured and singlet oxygen was detected by time- and spectrally resolved luminescence at regular time intervals. The irradiated samples were additionally investigated by HPLC, because UVA radiation can induce lipid peroxidation, ${ }^{23-25}$ even in the skin of mice $^{26}$ or cause mutations and DNA damage. ${ }^{27}$

\section{Results}

\section{Singlet oxygen generation in fatty acid solutions without UVA pre-irradiation}

Absorption at $355 \mathrm{~nm}$. Laser radiation at $355 \mathrm{~nm}$ was used to excite the investigated molecules. All fatty acids $\left(50 \mathrm{mmol} \mathrm{L}^{-1}\right)$ absorb partially UV radiation in the range from 200 to $250 \mathrm{~nm}$ (data not shown). Stearic acid solution without double bonds and oleic acid solution with only one double bond showed fairly low absorption at $355 \mathrm{~nm}$, and less than $0.5 \%$ of irradiation was absorbed at a concentration of $50 \mathrm{mmol} \mathrm{L}^{-1}$ for each fatty acids. For the other three fatty acid solutions the absorption ranged up to $5 \%$ (data not shown).

Singlet oxygen luminescence. The luminescence signals of the fatty acid solutions were measured time resolved at $1270 \mathrm{~nm}$ as well as spectrally resolved (1150-1400 nm). The signals were recorded in the range from $1150 \mathrm{~nm}$ to $1400 \mathrm{~nm}$ and all luminescence photons after the excitation laser pulse and noise $(120 \mu \mathrm{s})$ were summed up. Thus, a clear maximum of luminescence photons between 1270 and $1280 \mathrm{~nm}$ was detected for all fatty acids (data not shown). For singlet oxygen the transition to the ground state in ethanol is centered at $1274 \mathrm{~nm}$, this corresponds to an energy of $0.975 \mathrm{eV}$.

For stearic and oleic acid solution only a weak luminescence signal at $1270 \mathrm{~nm}$ was measured with a total photon number of 7700 and 11000 photons, respectively. These values are clearly higher than the measured luminescence at 1150 and
$1400 \mathrm{~nm}$ (4000 photons). The irradiation of fresh solutions of linoleic (29000 photons) and arachidonic (46000 photons) acids yielded a clear luminescence signal at $1270 \mathrm{~nm}$. In spite of the act a fresh linolenic acid solution was used, a total number of 126000 luminescence photons (about 3 times higher than the number of arachidonic acid) were detected, which might be caused by a higher content of already oxidized linolenic acid in the fresh solution.

Singlet oxygen generation in fatty acid solutions with UVA pre-irradiation. Since all fatty acid solutions showed a clear singlet oxygen signal under UVA irradiation, the generated singlet oxygen could in turn peroxide the unoxidized fatty acids and hence could change their molecular constitution. ${ }^{28}$ Consequently, the absorption of fatty acid solutions could be changed and oxygen could be consumed. Therefore, the different fatty acid solutions were irradiated with the broadband UVA-lamp for long time irradiation.

Changes in absorption spectra. The absorption spectra of the five fatty acid solutions after different irradiation times were recorded. Changes in the absorption spectra depended on the UVA irradiation time and are shown only for the excitation wavelength at $355 \mathrm{~nm}$ in Fig. 1. The maximal change in absorption was detected for arachidonic acid solution upon an irradiation time of 75 minutes, whereas the absorption of arachidonic acid solution increased up to $87 \%$. During irradiation, the oxygen concentration was kept constant by bubbling the solution with oxygen because the rate and the mechanism of such reactions depend on oxygen concentration. Due to the observed singlet oxygen generation and oxygen consumption during irradiation, the latter is discussed in a following paragraph, a change in the chemical molecule structure was supposed.

Changes in luminescence signals at $1270 \mathbf{~ n m}$. The luminescence signals (at $1270 \mathrm{~nm}$ ) of the five fatty acid solutions were detected at regular time intervals during UVA irradiation. The integrated singlet oxygen luminescence changed during irradiation with UVA, in particular for linolenic (3 double bonds) and arachidonic acid (4 double bonds) solution (Fig. 2A). The total count of luminescence photons increased for linoleic, linolenic and arachidonic acid solutions. For longer irradiation

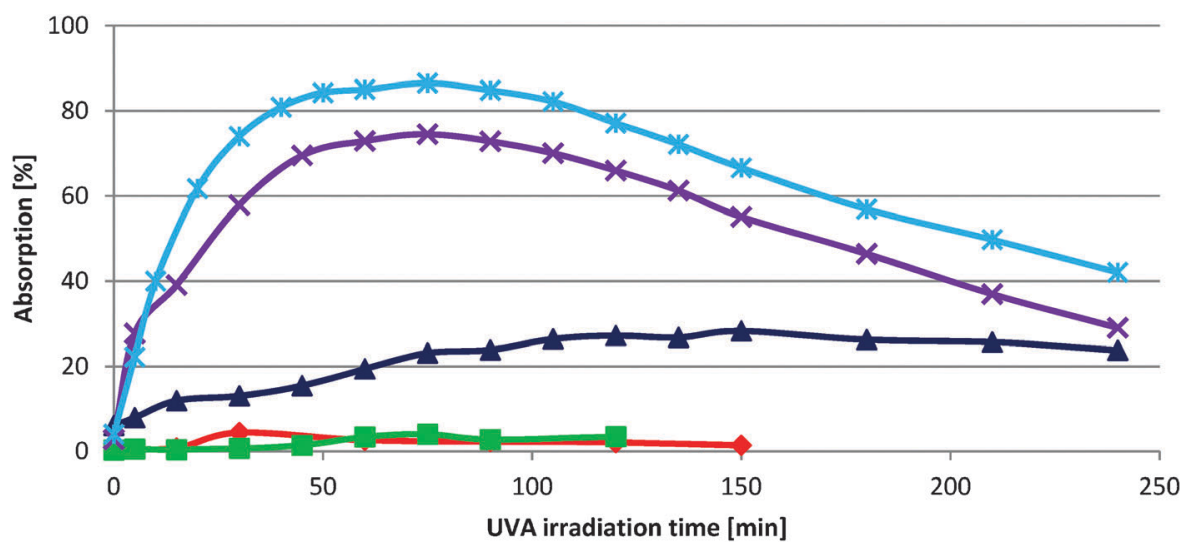

Fig. 1 The absorption value for all tested fatty acids $\left(50 \mathrm{mmol} \mathrm{L}^{-1}\right)$ at $355 \mathrm{~nm}$ after different irradiation times with $1 \mathrm{~W} \mathrm{~cm}^{-2}$ UVA radiation (red: stearic acid; green: oleic acid; dark blue: linoleic acid; purple: linolenic acid; cyan: arachidonic acid). 

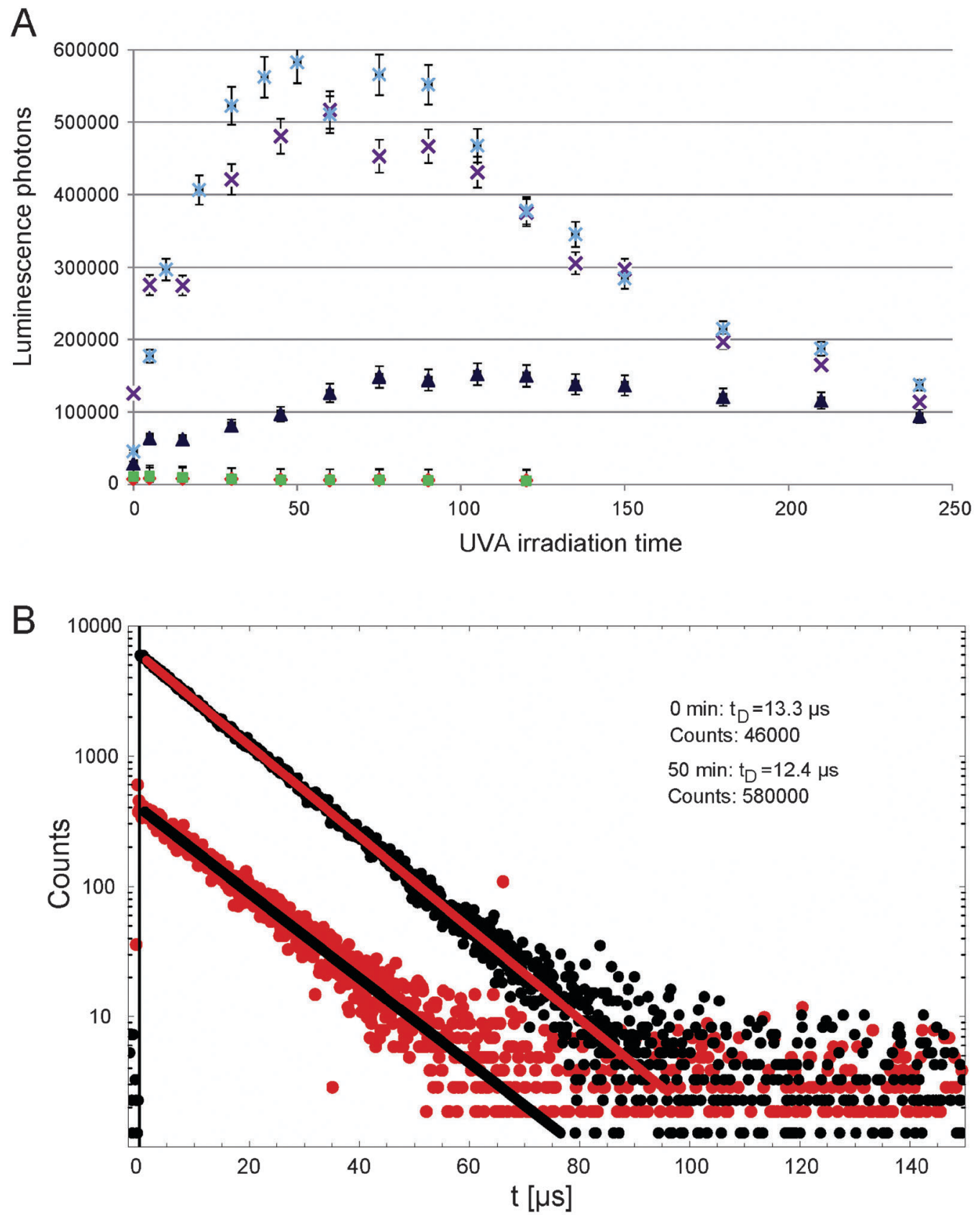

Fig. 2 The integrated signals of singlet oxygen luminescence at $1270 \mathrm{~nm}$ for all five fatty acids after different irradiation times with UVA radiation (A) (red: stearic acid; green: oleic acid; dark blue: linoleic acid; purple: linolenic acid; cyan: arachidonic acid; the values of stearic and oleic acid are magnified by a factor 10). Luminescence signals of $50 \mathrm{mmol} \mathrm{L}^{-1}$ arachidonic acid after 0 (red) and 50 (black) minutes continuous broadband UVA irradiation (B). Solid line is an exponential fit with Mathematica 5.2. The singlet oxygen decay time is in the range of $14 \pm 2 \mu \mathrm{s}$, the amount of photons increases with more than a factor of 12 .

times the count of luminescence photons decreased. For stearic and oleic acid solution the luminescence integral is pronounced for short irradiation times and decreases after 5 minutes of UVA-irradiation (Fig. 2A).

Fig. 2B exemplarily shows the luminescence signal of $50 \mathrm{mmol} \mathrm{L}^{-1}$ arachidonic acid solution before broadband irradiation and at the maximal luminescence after 50 minutes (black) UVA irradiation $\left(1 \mathrm{~W} \mathrm{~cm}^{-2}\right)$. The decay time slightly decreased but was still in the range of $14 \pm 2 \mu$ s. The total number of detected luminescence photons increased by a factor of more than 12
(46000 vs. 580000 photons). Table 1 shows the luminescence counts of ${ }^{1} \mathrm{O}_{2}$ photons detected at the beginning of UV irradiation for all fatty acid solutions, at the time of the maximal luminescence intensity and at the end of UV irradiation. The results show that the more double bonds the fatty acids have the more singlet oxygen luminescence is detected. The maximum values of luminescence increase from stearic acid solution (8600 counts), oleic acid solution (11 600 counts), linoleic acid solution (152 000 counts), linolenic acid solution (517000 counts), to arachidonic acid solution (583000 counts). 
Table 1 Singlet oxygen luminescence while longtime irradiation

Luminescence photons $^{a}$

Maximum of

Beginning of luminescence and End of UV

Fatty acid UV irradiation time of maximum irradiation (time)

\begin{tabular}{lrll}
\hline Stearic acid & 7700 & $8600(5 \mathrm{~min})$ & $5800(120 \mathrm{~min})$ \\
Oleic acid & 11000 & $11600(5 \mathrm{~min})$ & $5100(120 \mathrm{~min})$ \\
Linoleic acid & 29000 & $152000(105 \mathrm{~min})$ & $94500(240 \mathrm{~min})$ \\
Linolenic acid & 126000 & $517000(60 \mathrm{~min})$ & $114000(240 \mathrm{~min})$ \\
Arachidonic acid & 46000 & $583000(50 \mathrm{~min})$ & $137000(240 \mathrm{~min})$
\end{tabular}

${ }^{a}$ All detected photons within the measurement time span of ${ }^{1} \mathrm{O}_{2}$ luminescence $(120 \mu \mathrm{s})$.

Changes in the spectrally and time resolved luminescence signals. To clearly assign the detected signal at $1270 \mathrm{~nm}$ to singlet oxygen luminescence during the long time of UV irradiation, the luminescence signal was measured spectrally resolved in parallel to time resolution. Both results were combined to a three dimensional plot that clearly shows the increase and decrease of singlet oxygen luminescence over time. For arachidonic acid solution a clear maximum at $1270 \mathrm{~nm}$ after 50 minutes is noticeable (Fig. 3A). The noise level at 1150 and $1400 \mathrm{~nm}$ is nearly constant over time. For oleic acid solution a clear maximum at 5 minutes UVA irradiation is identifiable, after that the luminescence decreases to a noise level after 120 minutes of irradiation (Fig. 3B). Stearic acid solution showed a similar course of luminescence as oleic acid solution, whereas the luminescence intensity was small for all irradiation times (data not shown).

The maximum of luminescence for linoleic acid or linolenic acid solution was detected after 105 minutes or 60 minutes of irradiation, respectively, and for both, the signals decreased for irradiation times up to 120 minutes. As shown in Fig. 1 and 2A both the absorption and the luminescence increased to a maximum value at nearly the same time of broadband irradiation. This is consistent to the fact that absorption of photons is necessary to generate singlet oxygen. Based on the theory of singlet oxygen luminescence, that the total amount of luminescence clearly correlates with the concentration of singlet oxygen $\left[{ }^{1} \mathrm{O}_{2}\right] \cdot{ }^{29}$ Therefore, a correlation between $\left[{ }^{1} \mathrm{O}_{2}\right]$ and UVA absorption was made to prove whether the generation of singlet oxygen was caused by UVA absorption in fatty acid solutions (Fig. 4). The slope in Fig. 4 shows, within experimental accuracy, a good correlation of the increase of $\left[{ }^{1} \mathrm{O}_{2}\right]$ with increase of UVA absorption in fatty acids. The decrease of $\left[{ }^{1} \mathrm{O}_{2}\right]$ also correlates with UVA absorption but shows also a deviation for long UVA exposure times, indicating that the luminescence signal decreased faster than the absorption. This leads to the suggestion that molecules in the solution appear which absorb UVA but fail to generate singlet oxygen.

Oxygen consumption. To clarify the reason for the change in absorption the oxygen concentration in the fatty acid solutions was measured during broadband irradiation without oxygen bubbling. When starting with $100 \%$ oxygen saturation in the cuvette $\left(\left[\mathrm{O}_{2}\right]=1850 \mu \mathrm{mol} \mathrm{L}{ }^{-1}\right.$ for ethanol $)$, oxygen concentration clearly decreased with irradiation time and the decrease was correlated to the number of double bonds (Fig. 5). The more double bonds the faster was the oxygen decrease under UVA irradiation. The rate constants of the oxygen depletion of the five fatty acid solutions are shown in Table 2.

HPLC-MS measurement. Liquid chromatography analysis with mass spectroscopy detection was performed to confirm the observed results. The results showed that the molecular weight of fatty acids (except for stearic acid) increased by 32 Dalton that clearly indicates a binding of oxygen molecule to the fatty acids (hydroperoxides). Furthermore, dehydrated molecules could be detected (loss of $\mathrm{H}_{2} \mathrm{O}$ ).

For example, HPLC showed that the amount of arachidonic acid decreased with irradiation time (Fig. 6, red part). In contrast, the amount of hydroperoxides increased during the first 60 minutes and decreased afterwards (Fig. 6, blue part). HPLC-MS measurement showed that nearly no arachidonic acid or respective hydroperoxides were present after 240 min of irradiation time, but some other oxidized products (Fig. 6, green part).

\section{Discussion}

At first view, the absorption of radiation by fatty acids should be very low for wavelengths longer than $220 \mathrm{~nm} .{ }^{30}$ However, under atmospheric conditions, autooxidation of molecules can occur that cannot be avoided in the experimental setting. ${ }^{31,32}$ Autooxidized products of fatty acids and impurities may absorb radiation in the ultraviolet spectral range up to $400 \mathrm{~nm} .^{29}$ The results of luminescence detection clearly showed that UVA along with oxidized fatty acids generate singlet oxygen and the concentration of produced singlet oxygen increased with increasing double bonds of fatty acids solution. ${ }^{33}$

Skin is continuously targeted by UVA radiation, mainly due to solar radiation. In Germany, the annual terrestrial radiant exposure of UVA is about $22000 \mathrm{~J} \mathrm{~cm}^{-2}$ which is equivalent to $60 \mathrm{~J} \mathrm{~cm}^{-2}$ daily on average. ${ }^{34}$ A major constituent of the skin and its cells are various lipids containing the different fatty acids. In addition to living cells in the epidermis and dermis, the stratum corneum contains a mixture of ceramides and free fatty acids that forms the permeability barrier. ${ }^{35}$ Thus, fatty acids are a target of continuous UVA of solar radiation, which penetrates the skin down to the mid of the dermis at least.

Consequently, the five fatty acid solutions were exposed to continuous, broadband UVA radiation to investigate the impact of singlet oxygen generation. The results revealed an important process. The application of the continuous UVA radiation increased the absorption of fatty acid solutions including the wavelength $355 \mathrm{~nm}$ (Fig. 1). This wavelength is used in the experimental setup for luminescence detection of singlet oxygen. The change in absorption was fairly small for stearic and oleic acid, but substantially increased with irradiation time for fatty acid solutions with two or more double bonds. The absorption of linolenic or arachidonic acid solution reached values of about $20 \%$ within 5 min, which is equivalent to a radiant exposure of $300 \mathrm{~J} \mathrm{~cm}^{-2}$, the fivefold daily solar UVA radiation in Germany. ${ }^{34}$

Along with the increase of radiation absorption, the generation of singlet oxygen also substantially increased with irradiation time for linoleic, linolenic and arachidonic acid solution (Fig. 2). Singlet oxygen was continuously produced by UVA radiation up to $240 \mathrm{~min}$ 

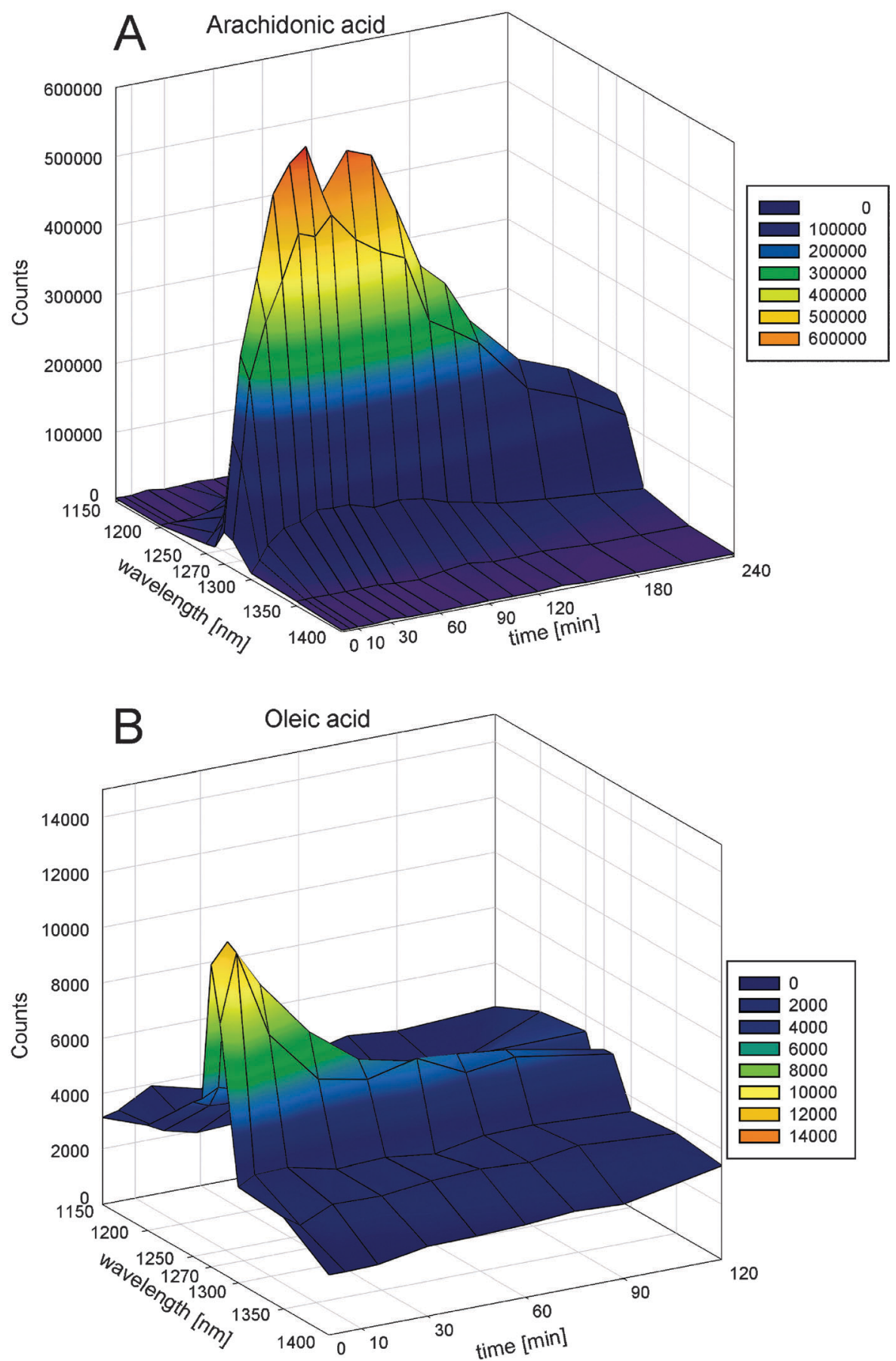

Fig. 3 Wavelength- and irradiation time-resolved three dimensional diagrams of arachidonic (A) and oleic acid (B). Values are determined by integration of the luminescence photons for each crossing point.

(e.g. for arachidonic acid), which was confirmed by spectral resolution (Fig. 3). At the same time, the oxygen concentration in solutions of fatty acid decreased, whereas the rate constant of oxygen consumption correlated to the number of double bonds (Fig. 5, Table 2). These results led to the assumption that singlet oxygen, produced by an interaction of UVA and peroxidized fatty acids, can react with other non-oxidized fatty acids.

Oxygen consumption occurred even for oleic acid solution and stearic acid solution but on a much longer time scale. This should be due to the less singlet oxygen production (oleic acid) or to the lack of double bounds (stearic acid). The consumption in stearic acid solution is most likely caused by impurities of the fatty acids (purity at about 99\%).

The oxygen consumption is obviously caused by the well-known reaction of singlet oxygen with unsaturated fatty acids. ${ }^{28,30}$ It is known that those oxidized fatty acids are the typical products being responsible for the so-called photo-oxidative stress signal in living cells. ${ }^{26,36}$ Our investigations with HPLC/MS showed a clear increase 


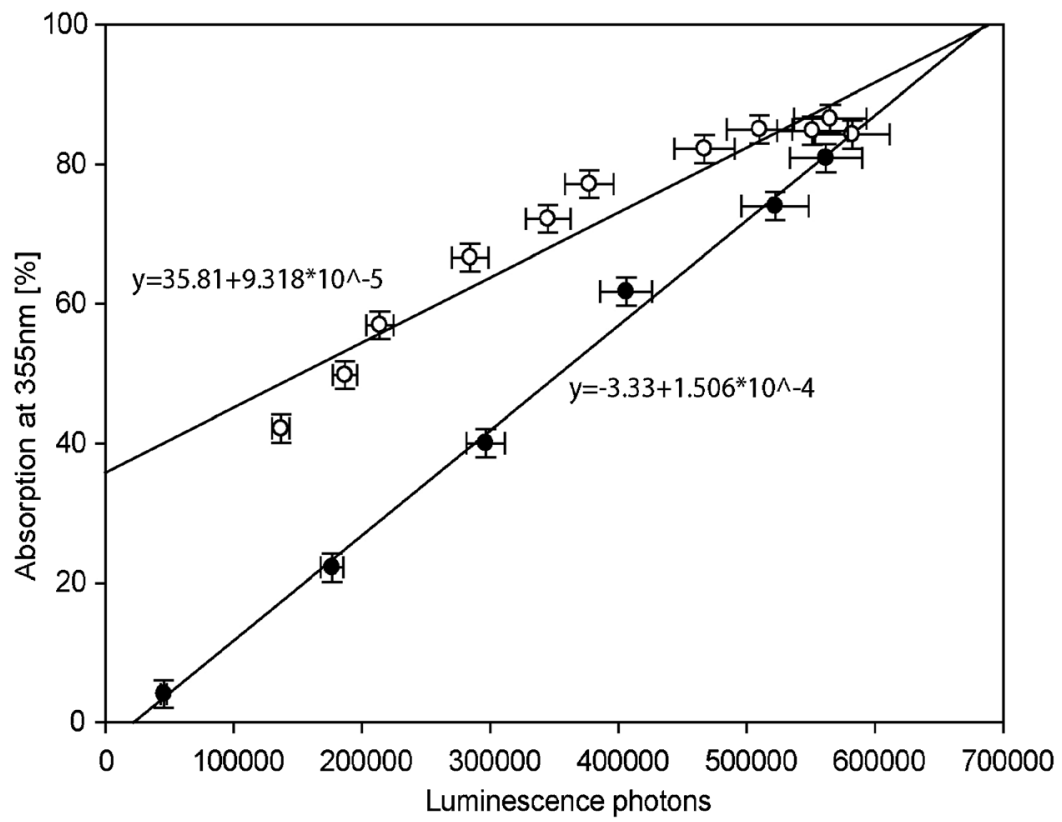

Fig. 4 Luminescence at $1270 \mathrm{~nm}$ vs. absorption at $355 \mathrm{~nm}$ for all measured points from 0 minutes up to 240 minutes of broadband UVA irradiation. For increase of absorption and luminescence a linear correlation could be shown.

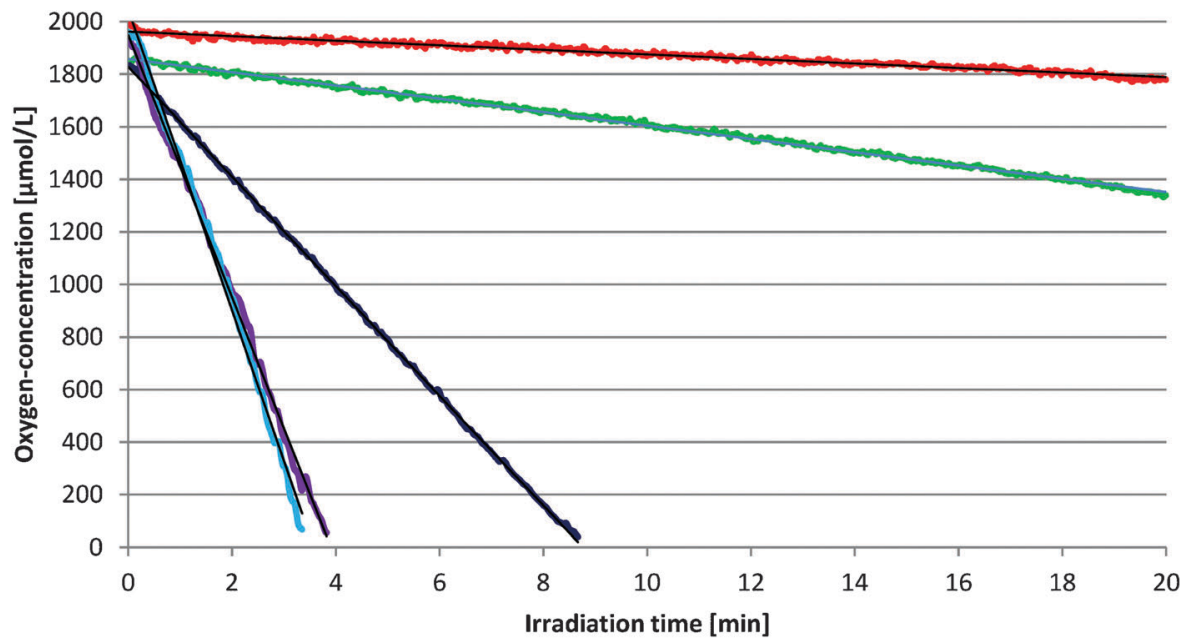

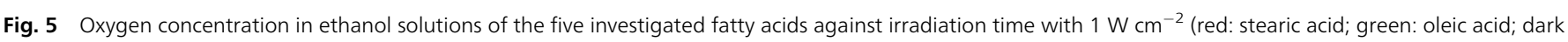
blue: linoleic acid; purple: linolenic acid; cyan: arachidonic acid).

Table 2 Oxygen consumption of fatty acids under UV irradiation

\begin{tabular}{ll}
\hline Fatty acid & Oxygen consumption rate $\left(\mu \mathrm{mol} \mathrm{s}{ }^{-1}\right)$ \\
\hline Stearic acid $18: 0$ & 0.15 \\
Oleic acid $18: 1$ & 0.42 \\
Linoleic acid $18: 2$ & 3.48 \\
Linolenic acid $18: 3$ & 8.32 \\
Arachidonic acid $20: 4$ & 9.55 \\
\hline
\end{tabular}

of molecular weight by 32 Dalton (molecular weight of $\mathrm{O}_{2}$ ) that is exemplarily shown for arachidonic acid solution in Fig. 6. With increasing irradiation time, the peak of peroxidized fatty acids in the diagram decreased. Based on this knowledge, the time course of UVA radiation in Fig. 3 should be explained as follows.
The singlet oxygen peroxidized fatty acids ${ }^{37}$ increased, which in turn led to higher UVA absorption and so to a higher amount of singlet oxygen. The 3D-plot shows that singlet oxygen luminescence decreases over time after having reached a maximum, which represents a decrease of the generated singlet oxygen due to further oxidation reactions with hydroperoxides.

To prove a correlation of singlet oxygen production and absorption of UVA radiation in fatty acid solutions, the total counts of luminescence were plotted against the UVA absorption at $355 \mathrm{~nm}$ for the different times of UVA irradiation (up to $240 \mathrm{~min}$ ). Except for stearic and oleic acid solution (low singlet oxygen production), the total count of luminescence was correlated to UVA absorption for the other fatty acid solutions. Thus, it can 


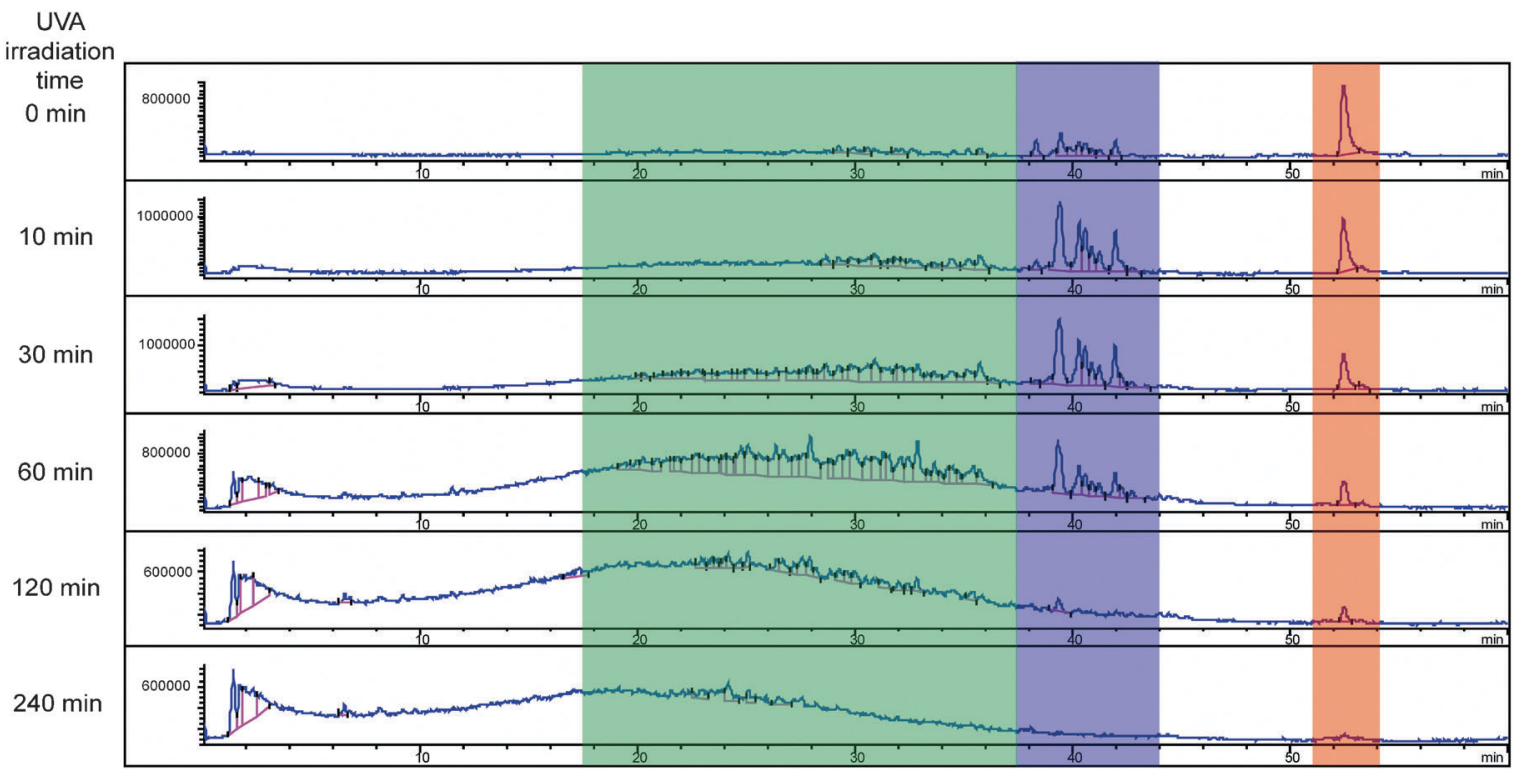

Fig. 6 HPLC analysis of arachidonic acid (red part: unoxidized arachidonic acid; blue part: molecules with $32 \mathrm{Da}\left(\mathrm{O}_{2}\right), 16 \mathrm{Da}(\mathrm{O})$ and14 $\mathrm{Da}\left(\mathrm{O}_{2}-\mathrm{H}_{2} \mathrm{O}\right)$ ) more molecular weight compared to arachidonic acid; green part: molecules with different weight ratios of oxygen content.

be assumed that the absorbing molecules (oxidized fatty acids) are responsible for the singlet oxygen luminescence. Furthermore for long exposure times, other oxidized products are generated in fatty acid solutions, which absorb UVA radiation but without being able to generate singlet oxygen (Fig. 4). Consequently, UV radiation is still absorbed but yielding less singlet oxygen.

It should be emphasized that in the case of UVA and fatty acid solutions, singlet oxygen is most likely not produced via normal photosensitizing because the typical chemical structures of photosensitizers (cyclic structures, conjugated double bonds) are lacking in fatty acids. The underlying mechanisms of singlet oxygen production in fatty acid solutions should be related to fast chemical reactions. This is confirmed by the fact that the time-resolved luminescence signals showed a rise time, which was close to the time resolution of the detection system and hence substantially shorter than for any known photosensitizer $(>0.2 \mu \mathrm{s})$. The decay time showed values of $14 \mu \mathrm{s}$, which is typical for singlet oxygen in ethanol solutions. ${ }^{38}$ Miyamoto et al. have already shown that lipid hydroperoxides (LOOH) can generate singlet oxygen and that these can decompose by heat, UV light and by the addition of transition metals. ${ }^{39}$ Furthermore Pratt et al. have shown that unstable hydroperoxides can lead to a propagation of lipid hydroperoxides as long as unoxidized fatty acids (LH) and oxygen are available. ${ }^{40}$ With the assumption that even in fresh solutions oxidized products of fatty acids are already present, which was confirmed by HPLC data in this study, the process actually start with a mixture of substances, even in fatty acids with a high purity. When exposed to UVA radiation, those molecules, particularly the oxidized ones, will undergo two different mechanisms. Firstly, these molecules might decompose to radicals and will react with surrounding molecules, to form new lipid radicals and lipid hydroperoxides. Both can in turn increase the overall number of lipid hydroperoxides again and again. Secondly, the generation of singlet oxygen is performed by the so-called Russell mechanism, ${ }^{41}$ whereby two hydroperoxides

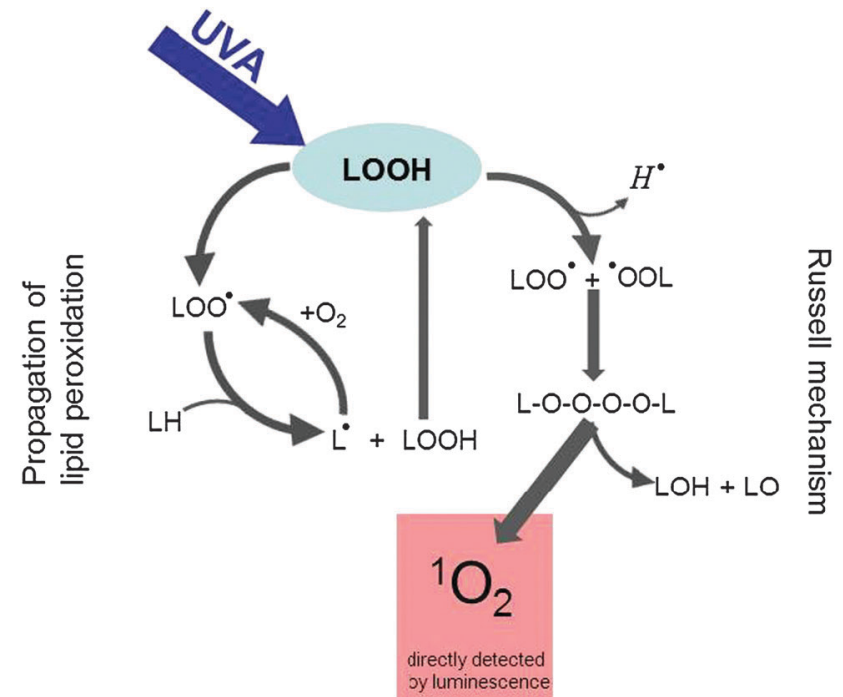

Fig. 7 Mechanism of singlet oxygen generation by UVA irradiation of lipid hydroperoxides and further oxidation of fatty acids.

link together to form a tetra-oxide. Consequently, ketones or aldehydes and singlet oxygen can be produced by cleavage of such tetra-oxides (Fig. 7). That description of singlet oxygen in those solutions under UVA exposure is not necessarily complete and may comprise other radical mechanisms.

\section{Experimental}

\section{Preparation of solutions}

The following substances were always freshly used in each single experiments and always dissolved in ethanol. All substances were purchased from Sigma-Aldrich (Steinheim, Germany) with a purity of about $99 \%$. Concentrations of stearic 
acid (18:0), oleic acid (18:1), linoleic acid (18:2), or linolenic acid $(18: 3)$ were $50 \mathrm{mmol} \mathrm{L}^{-1}$ each. The brackets show the ratio of the number of carbon atoms to the number of double bonds of the respective fatty acids.

\section{Transmission spectra}

Transmission spectra from $200-400 \mathrm{~nm}$ of each probe were recorded at room temperature with a Beckman DU640 spectrophotometer (Beckman Instruments $\mathrm{GmbH}$, Munich, Germany) using a quartz cuvette with an optical path of $1 \mathrm{~cm}$ (QS-101, Hellma Optik, Jena, Germany). The absorption values are calculated to be $A=100 \%-T$, where $T$ is the transmission value.

\section{Oxygen concentration in solution}

The oxygen concentration in solution was measured in the cuvette using a needle sensor that was placed in the cuvette (MICROX TX, PreSens GmbH, Regensburg, Germany). Thereby the cuvette was completely filled, hermetically closed and magnetically stirred during irradiation. The oxygen sensor continuously measured the oxygen concentration during irradiation.

\section{Luminescence experiments}

Fatty acids were transferred into a quartz cuvette (QS-101, Hellma Optik, Jena, Germany). They were excited using a frequency-tripled Nd:YAG laser (PhotonEnergy, Ottensoos, Germany) with a repetition rate of $2.0 \mathrm{kHz}$ (wavelength $355 \mathrm{~nm}$, pulse duration $70 \mathrm{~ns}$ ). The laser pulse energy for luminescence experiments was $50 \mu \mathrm{J}$. At regular time intervals, the singlet oxygen luminescence at $1270 \mathrm{~nm}$ was detected with using an IR-sensitive photomultiplier (R5509-42, Hamamatsu Photonics Deutschland GmbH, Herrsching, Germany) with a rise time of about 3 ns. Additional details of the setup were described elsewhere. ${ }^{42}$ The number of laser pulses for excitation was 60000 . The luminescence signal was detected at wavelengths of 1150, 1200, 1250, 1260, 1270 (emission maximum of singlet oxygen), 1280, 1300, 1350, $1400 \mathrm{~nm}$ using appropriate interference filters in front of the photomultiplier. All luminescence experiments are done in oxygen saturated solutions guaranteed by constant oxygen bubbling.

\section{Determination of singlet oxygen luminescence decay and rise time}

As shown in ref. 43 the luminescence intensity is given by

$$
I(t)=\frac{C}{\tau_{\mathrm{R}}^{-1}-\tau_{\mathrm{D}}^{-1}}\left[\exp \left(-\frac{t}{\tau_{\mathrm{D}}}\right)-\exp \left(-\frac{t}{\tau_{\mathrm{R}}}\right)\right]
$$

The constant $C$ was used to fit the luminescence signal. $\tau_{\mathrm{D}}$ and $\tau_{\mathrm{R}}$ are the decay and rise times, respectively. To determine the rise and decay times of singlet oxygen luminescence, we used the least square fit routine of Mathematica 5.2 (Wolfram Research, Berlin, Germany). The experimental error of the fit was estimated to be between 15 and $25 \%$ of the values that were determined by the fit. The low signal level in some samples yielded a higher error of $25 \%$. The integral of eqn (1) from $t=0 \rightarrow \infty$ yields the luminescence energy. To compare the luminescence energy of the different probes, the photomultiplier detected photons were added up from 100 ns after the laser pulse to the end of the luminescence signal.

\section{UVA irradiation}

Continuous, broadband UVA irradiation (320-400 nm) was performed using a high pressure mercury lamp (OmniCure Series 2000, IGB-Tech GmbH, Friedelsheim, Germany) at an intensity of $1 \mathrm{~W} \mathrm{~cm}^{-2}$. During irradiation the solutions were magnetically stirred and saturated with oxygen. The fatty acids were irradiated maximum for $240 \mathrm{~min}$, which is corresponding to a dose of $14.4 \mathrm{~kJ} \mathrm{~cm}^{-2}$.

\section{Liquid chromatography analysis and mass spectroscopy (HPLC-MS)}

The irradiated fatty acid samples were filtered using a PTFE filter (Chromafil, O-20/15, organic, pore size $0.2 \mathrm{~nm}$, MacheryNagel, Düren, Germany). A $100 \mu \mathrm{L}$ sample was analyzed using a 1100 HPLC (Agilent Technologies, Waldbronn, Germany) fitted with a C18 analytical column (Phenomenex Luna, particle size $3 \mu \mathrm{m}, 150 \times 2.00 \mathrm{~mm}$, Aschaffenburg, Germany) and a diode array detector (DAD). The data-files were analysed using a HPLC-3D-ChemStation Rev. B.03.01. For chromatography a gradient elution with water [0.0059 wt $\%$ trifluoroacetic acid] (solvent A) and acetonitrile (solvent B) at a constant flow rate of $1.0 \mathrm{~mL} \min ^{-1}$ was done. A gradient profile with the following proportions of solvent B was applied [ $t(\mathrm{~min}), \% \mathrm{~B}]:(0,5),(30,95)$. The chromatograms were monitored at $220 \mathrm{~nm}$. Mass spectroscopy was done using an Agilent 6100 Series Single Quadrupole Superior Line LC/MSD (Agilent Technologies, Waldbron, Germany) and an ESI/APCI Multimode Source.

\section{Conclusion}

We showed that fatty acids (with isolated double bonds) can generate singlet oxygen, although these molecules are not normal photosensitizers with conjugated double bonds or cyclic structures that offer a defined triplet $\mathrm{T}_{1}$ state. When pre-irradiated with UVA, the absorption of ultraviolet radiation substantially increased and in turn the production of singlet oxygen also increased. This vicious cycle rapidly consumes oxygen in solution leading to the production of peroxidized fatty acids.

These results in solutions can also occur inside cells, which are frequently exposed to ultraviolet irradiation as for the skin and the eye. Fatty acids are ubiquitously present in human cells but also exhibit the so-called "acid-mantle of the stratum corneum" that is important for both permeability barrier formation and cutaneous antimicrobial defense. ${ }^{44,45}$ Consequently, the generation of singlet oxygen may affect cellular and skin integrity leading to the frequently reported disturbance of the skin barrier, skin ageing and even skin malignancies.

The photosensitized generation of singlet oxygen by endogenous photosensitizers such as flavins and its role for those adverse reactions are well documented. ${ }^{19}$ In this study it could be shown that under UVA irradiation the amount of generated singlet oxygen depends on the number of double bonds of fatty acids and the amount of respective hydroperoxides. The extent of contribution 
to those adverse effects of singlet oxygen generation upon UVA exposure, in particular in the so-called "acid-mantle of the skin", is worth to be investigated in the near future.

\section{Acknowledgements}

The excellent technical assistance of Francesco Santarelli is gratefully acknowledged. Francesco Santarelli was funded by a grant of Dr August Wolff GmbH \& Co. KG. The work of Johannes Regensburger is supported by a grant of the 'Deutsche Forschungsgemeinschaft' (DFG), grant number RE3323/2-1. The work of Anita Gollmer is supported by a grant of the 'Deutsche Forschungsgemeinschaft' (DFG), grant number GO2340/1-1. The work of Alena Knak is supported by a grant of the 'Bayerische Eliteförderung'. All authors declare no conflict of interest.

\section{Notes and references}

1 S. Seite, A. Fourtanier, D. Moyal and A. R. Young, Br. J. Dermatol., 2010, 163, 903-914.

2 M. Berneburg and J. Krutmann, J. Photochem. Photobiol., B, 2000, 54, 87-93.

3 M. Berneburg, H. Plettenberg, K. Medve-Konig, A. Pfahlberg, H. Gers-Barlag, O. Gefeller and J. Krutmann, J. Invest. Dermatol., 2004, 122, 1277-1283.

4 A. M. Bode and Z. Dong, Sci. STKE, 2003, 2003, RE2.

5 L. O. Klotz, N. J. Holbrook and H. Sies, Curr. Probl. Dermatol., 2001, 29, 95-113.

6 L. O. Klotz, K. D. Kroncke and H. Sies, Photochem. Photobiol. Sci., 2003, 2, 88-94.

7 T. Schwarz, J. Photochem. Photobiol., B, 1998, 44, 91-96.

8 T. Schwarz, Keio. J. Med., 2005, 54, 165-171.

9 J. Wenk, P. Brenneisen, C. Meewes, M. Wlaschek, T. Peters, R. Blaudschun, W. Ma, L. Kuhr, L. Schneider and K. Scharffetter-Kochanek, Curr. Probl. Dermatol., 2001, 29, 83-94.

10 M. Wlaschek, I. Tantcheva-Poor, L. Naderi, W. Ma, L. A. Schneider, Z. Razi-Wolf, J. Schuller and K. ScharffetterKochanek, J. Photochem. Photobiol., B, 2001, 63, 41-51.

11 H. Ikehata and T. Ono, Photochem. Photobiol., 2007, 83, 196-204.

12 R. M. Tyrrell, Biochem. Soc. Symp., 1995, 61, 47-53.

13 S. L. Parker, T. Tong, S. Bolden and P. A. Wingo, Ca-Cancer J. Clin., 1997, 47, 5-27.

14 A. P. Schuch and C. F. Menck, J. Photochem. Photobiol., B, 2010, 99, 111-116.

15 E. Kvam and R. M. Tyrrell, Carcinogenesis, 1997, 18, 2379-2384.

16 G. M. Halliday, N. S. Agar, R. S. Barnetson, H. N. Ananthaswamy and A. M. Jones, Photochem. Photobiol., 2005, 81, 3-8.

17 K. Scharffetter-Kochanek, M. Wlaschek, P. Brenneisen, M. Schauen, R. Blaudschun and J. Wenk, Biol. Chem., 1997, 378, 1247-1257.

18 J. Baier, T. Maisch, M. Maier, M. Landthaler and W. Baumler, J. Invest. Dermatol., 2007, 127, 1498-1506.
19 J. Baier, T. Maisch, M. Maier, E. Engel, M. Landthaler and W. Baumler, Biophys. J., 2006, 91, 1452-1459.

20 J. Baier, T. Maisch, J. Regensburger, C. Pollmann and W. Baumler, J. Biomed. Opt., 2008, 13, 044029.

21 M. A. Lampe, M. L. Williams and P. M. Elias, J. Lipid. Res., 1983, 24, 131-140.

22 M. A. Lampe, A. L. Burlingame, J. Whitney, M. L. Williams, B. E. Brown, E. Roitman and P. M. Elias, J. Lipid. Res., 1983, 24, 120-130.

23 M. Wrona, W. Korytowski, M. Rozanowska, T. Sarna and T. G. Truscott, Free Radical Biol. Med., 2003, 35, 1319-1329.

24 T. Kriska, W. Korytowski and A. W. Girotti, Free Radical Biol. Med., 2002, 33, 1389-1402.

25 N. Bando, H. Hayashi, S. Wakamatsu, T. Inakuma, M. Miyoshi, A. Nagao, R. Yamauchi and J. Terao, Free Radical Biol. Med., 2004, 37, 1854-1863.

26 Y. Minami, K. Yokoyama, N. Bando, Y. Kawai and J. Terao, Free Radical Res., 2008, 42, 197-204.

27 T. M. Runger and U. P. Kappes, Photodermatol., Photoimmunol. Photomed., 2008, 24, 2-10.

28 A. W. Girotti, Photochem. Photobiol., 1990, 51, 497-509.

29 J. Baier, T. Maisch, J. Regensburger, M. Loibl, R. Vasold and W. Baumler, J. Biomed. Opt., 2007, 12, 064008.

30 O. Sergent, I. Morel, P. Cogel, M. Chevanne, M. Beaugendre, P. Chillard and J. Cillard, Anal. Biochem., 1993, 211, 219-223.

31 N. A. Porter, R. A. Wolf, E. M. Yarbro and H. Weenen, Biochem. Biophys. Res. Commun., 1979, 89, 1058-1064.

32 K. E. Fygle and T. B. Melo, Chem. Phys. Lipids, 1996, 79, 39-46.

33 W. Baumler, J. Regensburger, A. Knak, A. Felgentrager and T. Maisch, Photochem. Photobiol. Sci., 2012, 11, 107-117.

34 U. Feister, E. Jakel and K. Gericke, Photochem. Photobiol., 2002, 76, 281-293.

35 K. C. Madison, J. Invest. Dermatol., 2003, 121, 231-241.

36 A. W. Girotti, Free Radical Biol. Med., 2008, 44, 956-968.

37 A. W. Girotti, J. Photochem. Photobiol., B, 2001, 63, 103-113.

38 F. Wilkinson, W. P. Helman and A. B. Ross, J. Phys. Chem. Ref. Data, 1995, 24, 663-1021.

39 S. Miyamoto, G. E. Ronsein, F. M. Prado, M. Uemi, T. C. Correa, I. N. Toma, A. Bertolucci, M. C. Oliveira, F. D. Motta, M. H. Medeiros and P. D. Mascio, IUBMB Life, 2007, 59, 322-331.

40 D. A. Pratt, K. A. Tallman and N. A. Porter, Acc. Chem. Res., 2011, 44, 458-467.

41 G. A. Russell, J. Am. Chem. Soc., 1957, 79, 3871-3877.

42 J. Baier, M. Maier, R. Engl, M. Landthaler and W. Baumler, J. Phys. Chem. B, 2005, 109, 3041-3046.

43 D. Baumer, M. Maier, R. Engl, R. Markus Szeimies and W. Baumler, Chem. Phys., 2002, 285, 309-318.

44 J. W. Fluhr, J. Kao, M. Jain, S. K. Ahn, K. R. Feingold and P. M. Elias, J. Invest. Dermatol., 2001, 117, 44-51.

45 Y. Zheng, H. Yin, W. E. Boeglin, P. M. Elias, D. Crumrine, D. R. Beier and A. R. Brash, J. Biol. Chem., 2011, 286, 24046-24056. 